\title{
Getting your Ys crossed
}

The mammalian Y chromosome (chrY) has an unusual evolutionary history that has endowed it with similarly unusual features: the malespecific region (MSY), which does not ordinarily recombine with the X chromosome, composes $~ 95 \%$ of chrY and contains massive palindromic sequences. Although it has been proposed that palindromes are maintained by gene conversion, how this happens is poorly understood. A study in humans now provides evidence that the repair of DNA double strand breaks (DSBs) might be responsible for maintenance - a mechanism that has implications for human fertility.

Each chrY palindrome consists of two mirror-image arms separated by a short spacer. Lange and colleagues suggested that gene conversion in palindromes might occur by noncrossover homologous recombination between $Y$ sister chromatids (see figure). Based on what is known about homologous recombination in general, they predicted that occasionally crossover resolution would also occur. Crossover between Y sister chromatids could produce a mirrorimaged chrY with two centromeres and with the axis of symmetry at the site of recombination (see figure) this is known as an isodicentric $\mathrm{Y}$ chromosome (idicY).

The palindrome arms contain genes that are important for spermatogenesis; could the formation of idicY therefore be a cause of male infertility? The authors used samples from 2,380 patients with spermatogenic failure, chrY anomalies or a sex-reversal phenotype (in which the patient is anatomically feminine) to look for evidence of the mechanism of gene conversion. They screened the samples using PCR for chrY markers and found 51 cases with idicY (49 of which were idicYp). They then used fluorescence in situ hybridization (FISH), including high-resolution FISH on interphase chromosome spreads, to work out the details of how the idicYs had formed. The patterns of hybridization confirmed the authors' predicted model that the idicYs are formed by homology-mediated crossing over between opposing palindrome arms on sister chromatids. The observations supported their hypothesis that DSBs in the MSY are repaired by homologous recombination, normally with non-crossover resolution that maintains the palindromes but occasionally by crossover resolution that creates an abnormal chrY.

So what are the consequences of idicY formation? The frequency of idicY in their patient panel led the authors to suggest that this type of abnormality is likely to be one of the most common genetic causes of spermatogenic failure in otherwise healthy men. For example, idicYp chromosomes lack distal Yq genes that are needed for spermatogenesis. The group of patients with idicY also included individuals with sex reversal. As an idicYp chromosome includes two copies of the male sex determination gene SRY, this was surprising. The authors found that idicY chromosomes are somatically unstable - particularly those chromosomes with a long intercentromeric distance (which were more common in individuals with sex reversal). They found that this mitotic instability results in 46,X,idicYp individuals being mosaic for 45,X cells. Consistent with this observation, five patients with idicYp had the clinical features of Turner's syndrome, which is commonly associated with a 45 , X karyotype. Therefore, the authors suggest that sex reversal and Turner's syndrome can arise as an indirect consequence of abnormal recombination between chrY palindromes.

Although the mechanism of non-crossover homologous recombination successfully maintains chrY structure and function, the recombinogenic nature of the palindromes creates an 'Achilles heel' in the form of crossover events, which can have significant clinical consequences for fertility and development. Screening for idicY chromosomes might help to explain many more cases of spermatogenic failure.

Mary Muers

ORIGINAL RESEARCH PAPER Lange, J. et al.

Isodicentric $Y$ chromosomes and sex disorders as byproducts of homologous recombination that maintains palindromes. Cell 138, 855-869 (2009) 